\title{
Supporting Information: \\ Ubiquity of Exciton Localization in Cryogenic Carbon Nanotubes
}

\author{
Matthias S. Hofmann, ${ }^{\dagger}$ Jonathan Noé, ${ }^{\dagger}$ Alexander Kneer, ${ }^{\dagger}$ Jared J. Crochet, ${ }^{\ddagger}$ \\ and Alexander Högele ${ }^{\star, \dagger}$
}

\author{
${ }^{\dagger}$ Fakultät für Physik, Munich Quantum Center, and Center for NanoScience (CeNS), \\ Ludwig-Maximilians-Universität München, Geschwister-Scholl-Platz 1, D-80539 München, \\ Germany \\ ${ }^{\ddagger}$ Physical Chemistry and Applied Spectroscopy Division, Los Alamos National Laboratory, \\ Los Alamos, New Mexico 87545, United States \\ ^ corresponding author. E-Mail: alexander.hoegele@lmu.de
}

\begin{abstract}
Complementary to Figure 2 of the main manuscript, Figures S1, S2, and S3 present additional photoluminescence (PL) spectra (left panels), the extracted PL peak positions (triangles in the right panels) and the theoretical temperature dependence of the band gap according to Ref. 1 (black solid line in the right panels) of individual HiPco, CoMoCAT and as-grown carbon nanotubes (CNTs), respectively, at 295, 77, and $4.2 \mathrm{~K}$. Peaks marked by the asterisks correspond to CNT-related Raman bands and were not considered for analysis of the multipeak PL structures. To expand the set of presented chiralities from CoMoCAT and as-grown CNTs in Figure 3 of the main manuscript, Figures S2 and S3 also show exemplary PL spectra of $(8,3)$ CoMoCAT nanotubes as well as of $(6,5),(7,3)$, and $(8,3)$ as-grown CNTs, respectively.
\end{abstract}

\section{References}

[1] Capaz, R. B.; Spataru, C. D.; Tangney, P.; Cohen, M. L.; Louie, S. G. Phys. Rev. Lett. 2005, 94, 036801. 

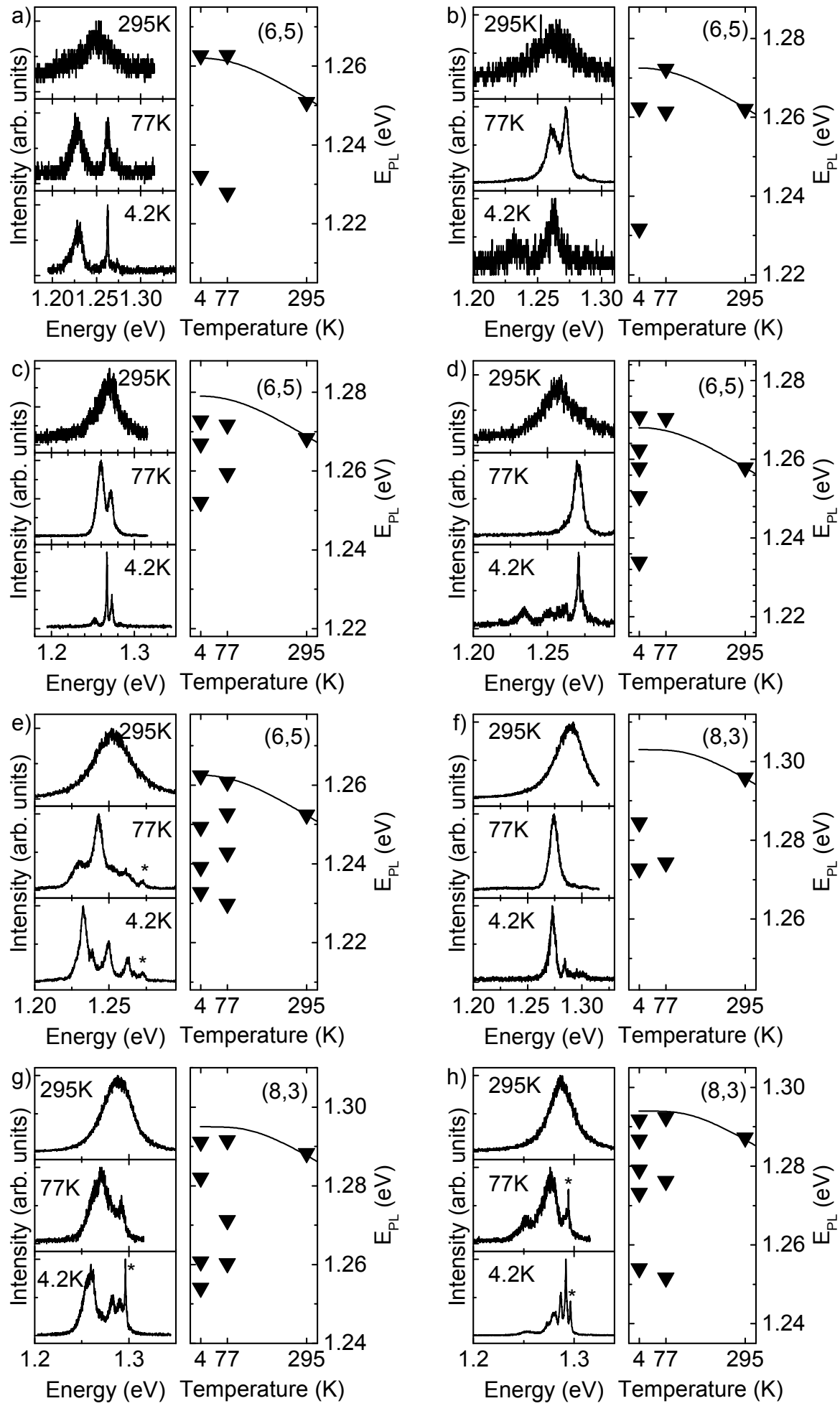

Figure S1: (a-h) Photoluminescence spectra (left panels) and peak emission energies $E_{\mathrm{PL}}$ (right panels) of individual HiPco carbon nanotubes at 295, 77, and $4.2 \mathrm{~K}$. The energy dispersion with temperature (black solid line in the right panels) shows the theoretical dependence of the band gap according to Ref. 1 . 

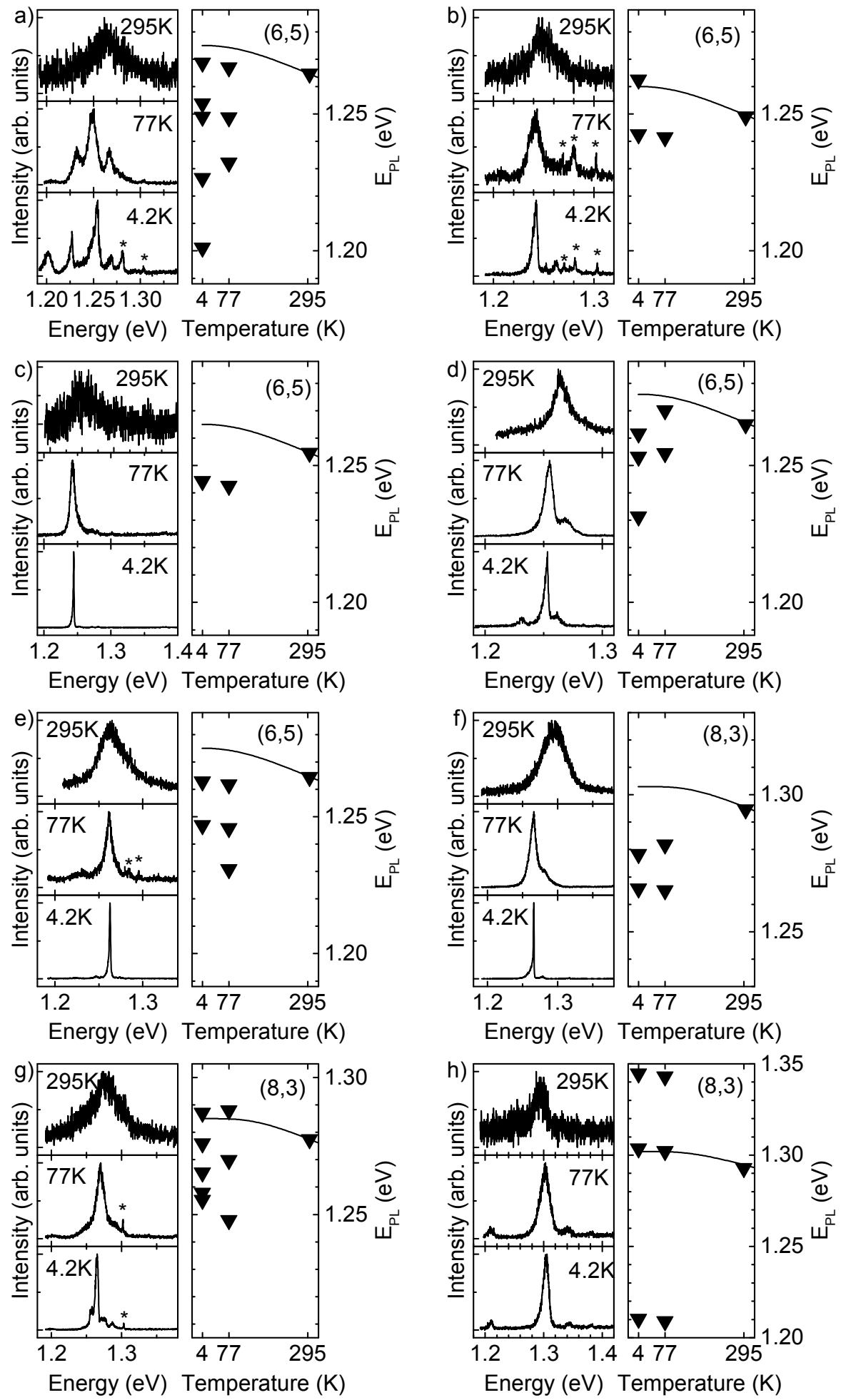

Figure S2: (a-h) Photoluminescence spectra (left panels) and peak emission energies $E_{\mathrm{PL}}$ (right panels) of individual CoMoCAT carbon nanotubes at 295, 77, and $4.2 \mathrm{~K}$. The energy dispersion with temperature (black solid line in the right panels) shows the theoretical dependence of the band gap according to Ref. 1 . 

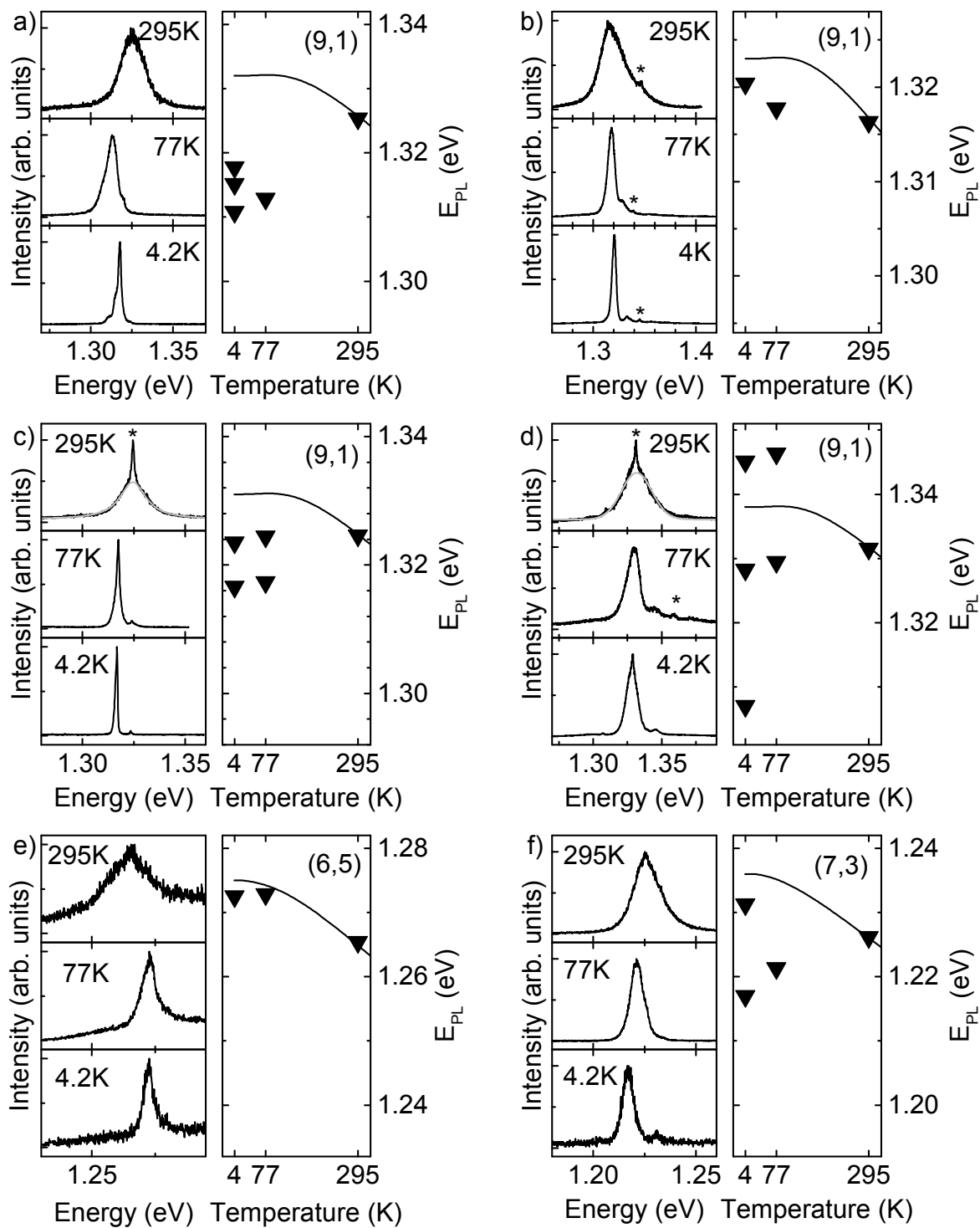

Energy (eV) Temperature (K)
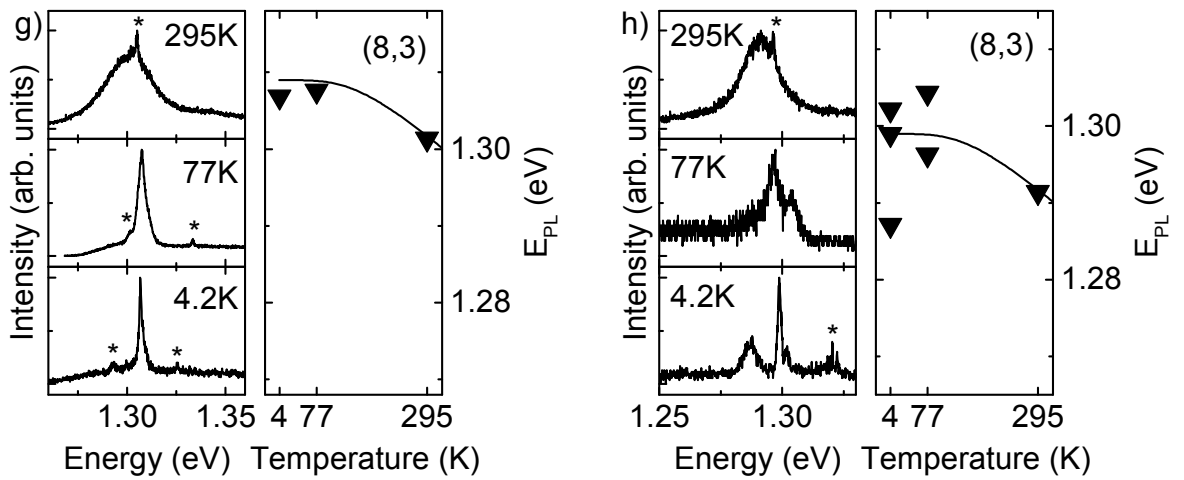

Figure S3: (a-h) Photoluminescence spectra (left panels) and peak emission energies $E_{\mathrm{PL}}$ (right panels) of individual as-grown carbon nanotubes at 295, 77 , and $4.2 \mathrm{~K}$. The energy dispersion with temperature (black solid line in the right panels) shows the theoretical dependence of the band gap according to Ref. 1 . 